\title{
Update on Interventions to Prevent or Reduce Frailty in Community-Dwelling Older Adults: a Scoping Review and Community Translation
}

\author{
Xiao Liu ${ }^{1} \cdot$ Daniella Hui-Min $\mathrm{Ng}^{1} \cdot$ Jean Wei-Ting Seah ${ }^{1} \cdot$ Yasmin Lynda Munro $^{2} \cdot$ Shiou-Liang Wee $^{1,3}$ (D)
}

Published online: 22 April 2019

(C) The Author(s) 2019

\begin{abstract}
Purpose of Review With increasing interest in frailty prevention and management globally, this scoping review provides an update to the current knowledge on interventions to reduce frailty in community-dwelling older adults and its state of translation in real-world settings.

Recent Findings Updating previous findings of 14 studies from 2000 to 2016 by Puts et al., we reviewed 10 additional studies from 2016 to 2018 and found that up to date, 18 of 24 studies overall showed effective interventions in preventing or reducing frailty (as the intervention target) in community-dwelling older adults. Multidimensional frailty definitions and a multi-domain approach have been increasingly employed for frailty identification and intervention. The effective interventions included various modalities of exercise training, nutrition education/program, cognitive training, geriatric assessment, and management coupled with psychosocial interventions/support that may sustain program participation. While resistance training was the most commonly employed modality of physical training, we did not find evidence that it is more effective than other modalities. Effective interventions can be delivered in a primary care or other community settings by multi-disciplinary combination of professionals and volunteers. There has been better recognition of the need for community translation in frailty reduction in the developed countries, with initial policy efforts introduced in some countries.

Summary To further the evidence-based and community translation, well-designed studies aimed at maximizing function and promoting aging in place using multi-domain interventions are essential. This will better inform comprehensive policies to address frailty prevention/reduction through appropriate health and social programs/services provision and financing.
\end{abstract}

Keywords Frailty $\cdot$ Older adults $\cdot$ Resistance training $\cdot$ Scoping review $\cdot$ Community translation

Xiao Liu and Daniella Hui-Min Ng contributed equally to this work.

This article is part of the Topical Collection on Frailty in the Elderly

Electronic supplementary material The online version of this article (https://doi.org/10.1007/s13670-019-0277-1) contains supplementary material, which is available to authorized users.

Shiou-Liang Wee

weeshiouliang@gmail.com

1 Geriatric Education and Research Institute, 2 Yishun Central 2, Singapore 768024, Singapore

2 Medical Library, Lee Kong Chian School of Medicine, Singapore, Singapore

3 Health Services and System Research Program, Duke-National University of Singapore Medical School, Singapore, Singapore

\section{Introduction}

Frailty has drawn growing interest as a clinical geriatric syndrome with its increasing prevalence in older populations due to its associations with hospitalization rates, functional decline, long-term care needs, and death $[1,2]$. Estimates suggest that $5-16 \%$ of community-dwelling adults in developed countries are frail [3, 4], with more (pooled prevalence 49\%) in the transitional pre-frail state [5]. Recent reviews concluded that frailty can be reversed $[6 \bullet \bullet, 7 \bullet]$, and interventions that significantly postponed or reduced frailty include physical activities alone, or in combination with nutritional and/or cog-

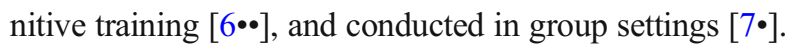

Most research studies assessed frailty using the Fried et al. physical frailty phenotype [1] (presence of weakness, slow gait, exhaustion, unintended weight loss, and low physical activity: $1-2$ Pre-frail, $\geq 3$ Frail) as it provides more consistent 
prevalence estimates. However, there is emerging evidence for a multi-dimensional (physical, cognitive, social, and psychological) concept of frailty $[8,9 \bullet]$, with implications for multi-domain type of interventions for frailty.

The World Health Organization's (WHO) [10] strategy for healthy aging has shifted emphasis from longevity to physical and cognitive functioning. Given the increasing prevalence of frailty with age, appropriate actions to reduce frailty, disability, and dependence among older adults should be at the forefront of all policies to tackle the challenges of an aging population. While it may pose a significant challenge and burden, it also presents opportunities for concerted action to foster effective policies and initiatives in community frailty prevention and reduction.

Three previous reviews on interventions to prevent or reduce frailty in community-dwelling older adults were constrained by the limited number of articles that were published. A review by Puts et al. [6••] included 14 studies between January 2000 and February 2016 on interventions to prevent or reduce frailty in communitydwelling older adults. Published in the same year, Frost et al. [11•] reviewed 10 studies from January 1990 to May 2016 on randomized controlled trials (RCT) and crossover trials of health promotion interventions for community-dwelling older people with mild/pre-frailty. Additionally, Apóstolo et al. [7•] reviewed 21 studies from January 2001 to November 2015 that included community-dwelling older people, with the addition of studies from hospitals and eldercare institutions, which were excluded by Puts et al. [6••].

This paper provides an update to previous reviews of 14 papers from 2000 to 2015 by Puts et al. [6••] by incorporating more recent publications of 10 papers from January 2016 to July 2018. Furthermore, we also searched for evidence on any successful community dissemination and/or implementation of interventions and population strategies to reduce frailty. While the prevention/reversal of frailty would be an invaluable benefit to our aging society, it is paramount to effectively translate the trials' results using available resources in the real-world settings. Indeed, a great challenge facing the global health community is the dissemination and implementation of proven interventions and its translation in the community on a broad scale.

\section{Methods}

\section{Review Methodology}

To update the findings by Puts et al. [6••], we followed the same scoping review methods described in their paper. Briefly, we used the methodological framework of Arksey and O'Malley [12] as a guide, with modifications [13]. We systematically searched electronic databases and gray literature to identify interventions and policies that aimed to prevent or reduce the level of frailty. Following the guidelines for PRISMA statement [14], the scoping review model included (i) identification of research question, (ii) identification of relevant studies, (iii) selection of studies, (iv) charting of data, (v) summary and report the results, and (iv) consult or survey stakeholders to validate/inform study findings.

\section{Search Methods}

Studies were included based on the following criteria: (i) original publications of interventions to prevent or reduce the level of frailty in community-dwelling older adults aged $\geq 65$ years; (ii) or studies that included a wider age range were eligible if the mean/median age of the study population was aged $\geq$ 65 years; (iii) or if they have included a subgroup analysis for the population aged $\geq 65$ years. Only studies that provided a measure of frailty before and after the intervention were included. Gray literature that had information on the (i) dissemination and/or implementation of interventions or (ii) policies to prevent/reduce frailty were also included.

We devised a core search strategy in Ovid MEDLINE and adapted it for other databases using the appropriate syntax (Appendix A). We limited our searches to publications on humans, in English language only with date from January 2016 to July 2018.

We searched the following electronic databases: Ovid MEDLINE(R), EMBASE, Cochrane Central Register of Controlled Trials (CENTRAL), Cumulative Index to Nursing and Allied Health (CINAHL), PsycINFO, AgeLine, Sociological Abstracts, Web of Science, Health Technology Assessment (Canadian Search Interface), and the reference list of included studies. In addition, we hand-searched Google Scholar and key geriatric medicine and public health journals to identify studies that may not be identified by the databases.

A search for gray literature was carried out in August 2018 from the following resources: World Health Organization [10], New York Academy of Medicine Gray Literature Report [15], Open Gray [16], and Science.gov [17] using keywords such as "frailty" and "frail elderly" and limited to publications from January 2000 to July 2018. Our inclusion criteria were reports by healthcare or health policy organizations and OpenGrey that had information on dissemination, implementation of, and/or policy on frailty interventions. Due to limited gray literature, we also conducted a short survey of participating international keynote speakers at an International Conference on Frailty at Shanghai on 3 September 2018 [18] on the extent of implementation of, and/or policy on frailty intervention in respective regional/country. 


\section{Study Selection}

We determined the eligibility of studies by using the Covidence systematic review software [19], to review abstracts based on the inclusion and exclusion criteria. Two authors independently selected the abstracts, with a third author's decision when conflicts occurred. Full-text articles were obtained for selected studies, and abstracts that provided insufficient details for acceptance. Studies that included frail community-dwelling older adults were excluded if they did not examine frailty-specific outcomes.

The information abstracted included characteristics of the study population, study design, frailty assessment used, levels of frailty of study participants, intervention details and outcomes, and analyses used (Tables 1,2, and 3). We assigned each paper a unique identification number to conveniently and clearly track relevant studies [20].

\section{Quality Assessment}

The quality of the included studies was assessed using the Mixed Methods Appraisal Tool (MMAT) [21], but none were excluded based on the score.

\section{Data Analysis}

The results are summarized using a narrative descriptive synthesis approach rather than a pooled analysis approach, which was not appropriate due to heterogeneity in study inclusion criteria, interventions, frailty assessment tools, and/or outcomes.

\section{Results}

\section{Characteristics of Included Studies}

In this review, 2385 abstracts were retrieved. After removal of duplicates, 1652 abstracts were each independently reviewed by two authors. Sixteen papers were selected for full-text assessment of eligibility, and 10 papers were eventually included in qualitative synthesis (Fig. 1). Seven studies employed randomized controlled trials (RCTs) [22-28], two were singlearm feasibility studies [29,30], and one employed a cluster non-randomized controlled approach [31] (Tables 1, 2, and 3, Appendix B).

Five studies were conducted in Asia [23, 26, 27, 29, 31], four in Europe [22, 24, 25, 28], and one in Canada [30]. The number of participants in the single-arm studies was nine [29] and 51 [30], respectively. Among the other eight studies, the number of participants ranged from 41 [26] to 459 [22] with a mean of 183 participants and a total of 1461 participants. The study mean/median age ranged from $71.6[23]$ to 86 years
[22], and percentage women study ranged between 31.2 [27] and $90.2 \%$ [26] (Table 1, Appendix B).

\section{Quality of the Included Studies}

The quality assessment results are presented in Appendix B, Table C. The quality was moderate to good for all the studies, although the information about the target population and attempts to achieve the population representativeness were not clearly described in three non-RCTs [29-31]. The randomization method was not reported for one RCT [24], and a relatively small control group size in another RCT [22]. Additionally, outcome assessors were not blinded in two of the RCTs [26, 28] and not reported in two RCTs [24, 25]. Adherence to the intervention sessions was lower than $80 \%$ in three studies $[23,24,28]$.

\section{Interventions Studied}

Frailty definitions and characteristics of the participants are listed in Table 1. Eight distinct assessments for frailty were used across 10 studies, with two studies $[23,30]$ that used two distinct frailty assessments each. Other than the most commonly used Fried physical phenotype [1] by four studies $[23,24,26,28]$, the other studies have adopted frailty definition/assessment that also included cognition and/or social dimensions $[22,27,30]$, and/or checklist/questionnairetype assessment [27, 29, 31], with less need of measurement instruments (e.g., grip strength and gait speed) for easy screening and identification. Four studies [22, 27, 30, 31] used multidimensional frailty definitions, with corresponding multidomain $[27,31]$ or multi-disciplinary $[22,30]$ interventions.

The characteristics of interventions are detailed in Table 2. Two studies comprised of physical education alone [26, 29], while the remaining eight studies were multi-domain in nature, including (i) physical exercise with nutrition $[25,28]$ alone; (ii) physical exercise with nutrition coupled with (a) psychosocial intervention [23, 27, 31], or (b) prescription and social interventions [24]; and (iii) involving multidisciplinary professional expertise $[22,30]$. Of eight controlled studies, the control/comparator groups received interventions that comprised of usual daily activities [27], preventive home visit [22], usual services by urban districts [22], low-level care of a single education session [23], routine primary care [24, 28], cognitive training with social contact [25], resistance training [26], and physical exercise with nutrition [31].

The physical exercise interventions were performed in groups $[23,26,27,29,31]$ or individually at home $[24,25$, $28]$ with sessions ranging in frequency from once weekly [31] to 4 days per week $[24,28]$. Exercises emphasized one or more components of endurance, strength, power, mobility, balance, and/or coordination. Nutrition interventions included nutrition assessment [28], personal nutrition guidance and 


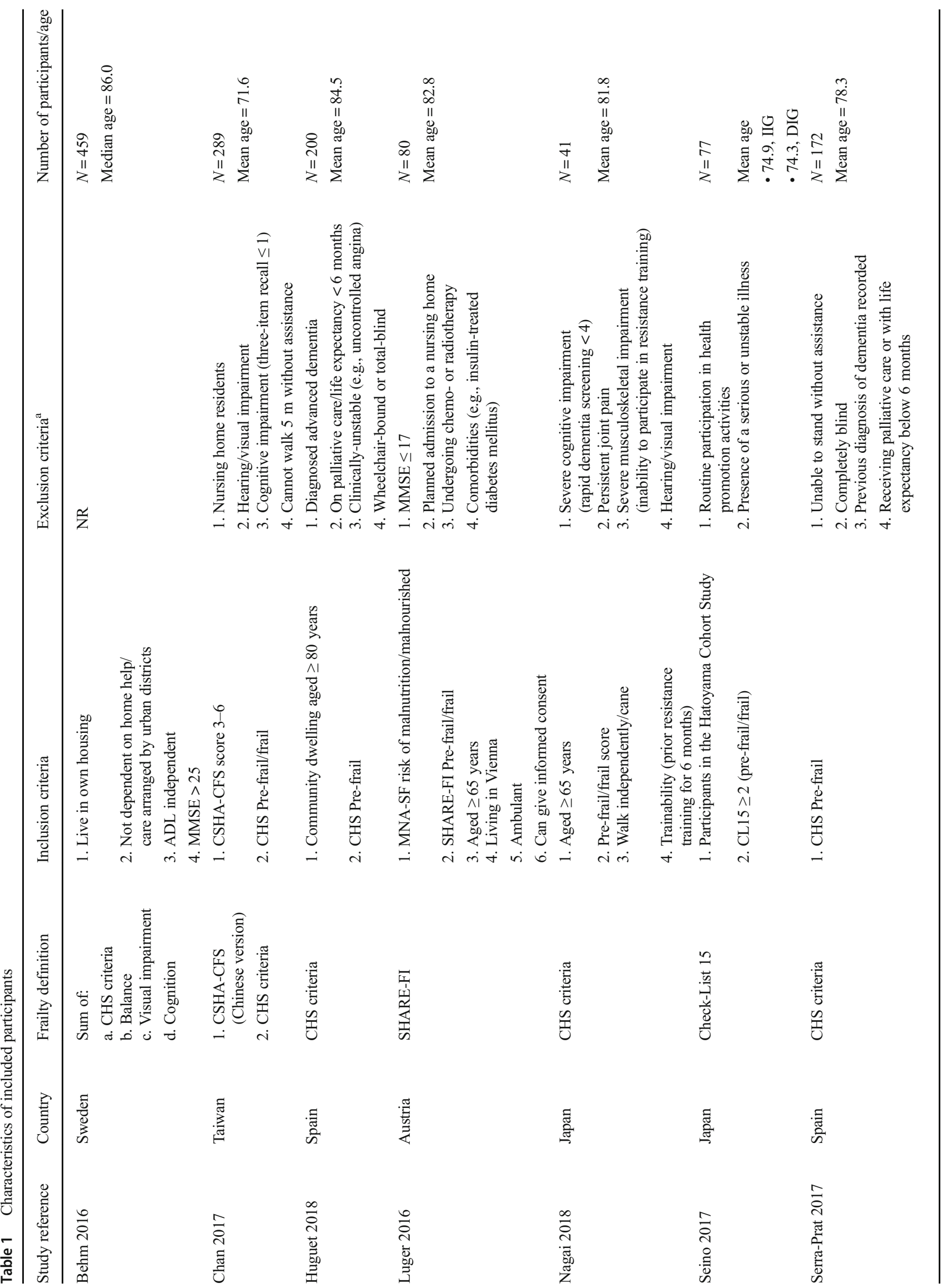




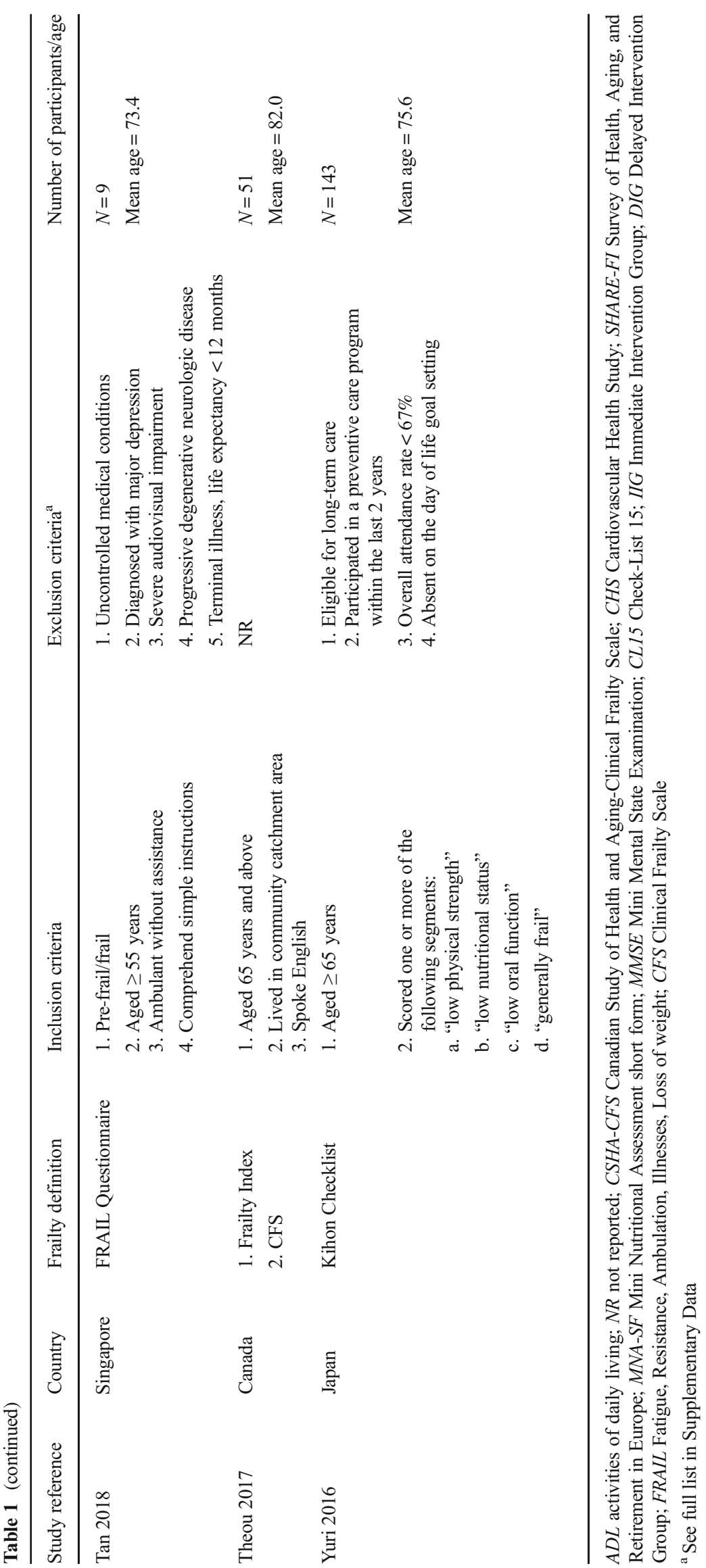




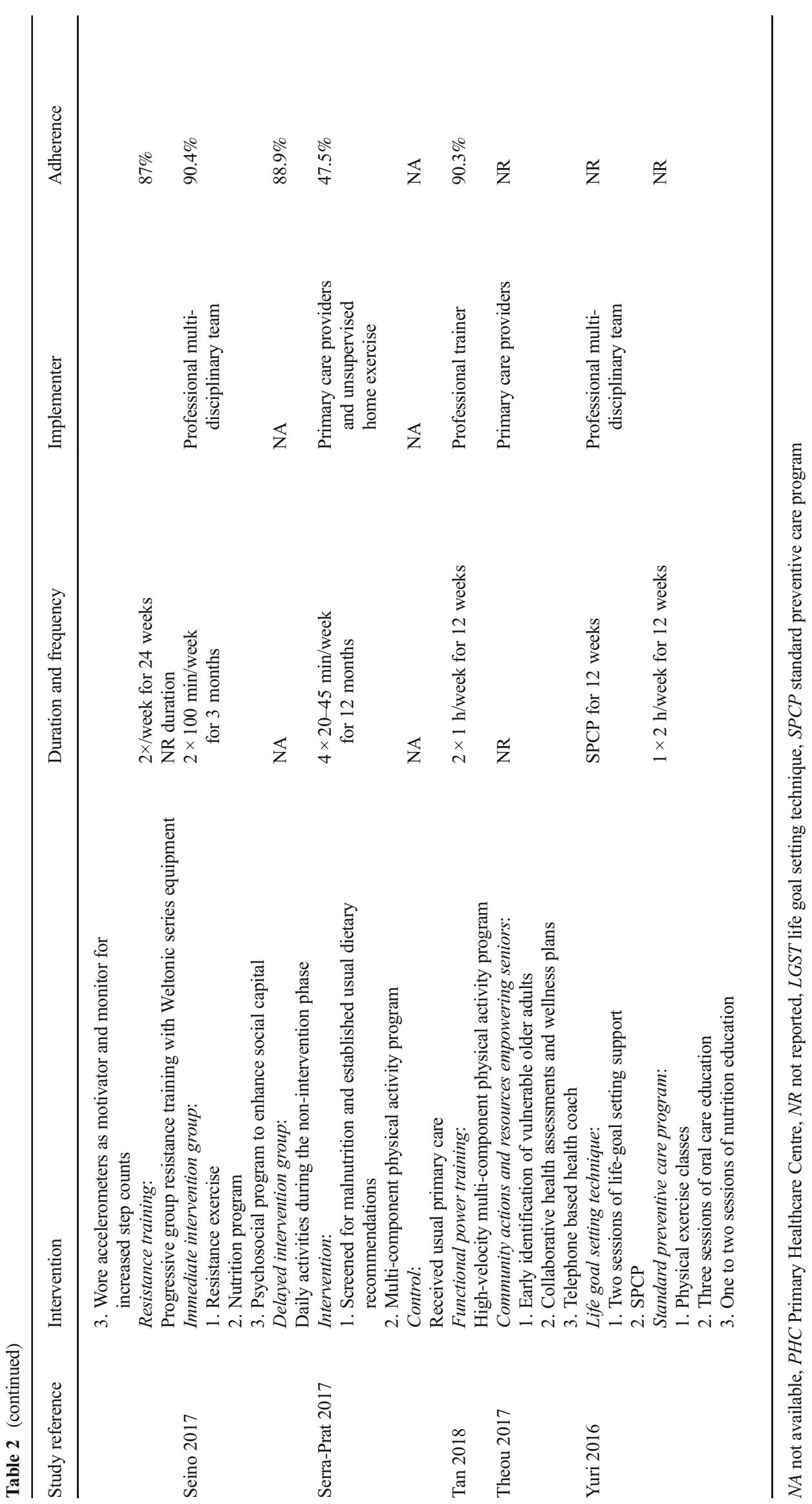




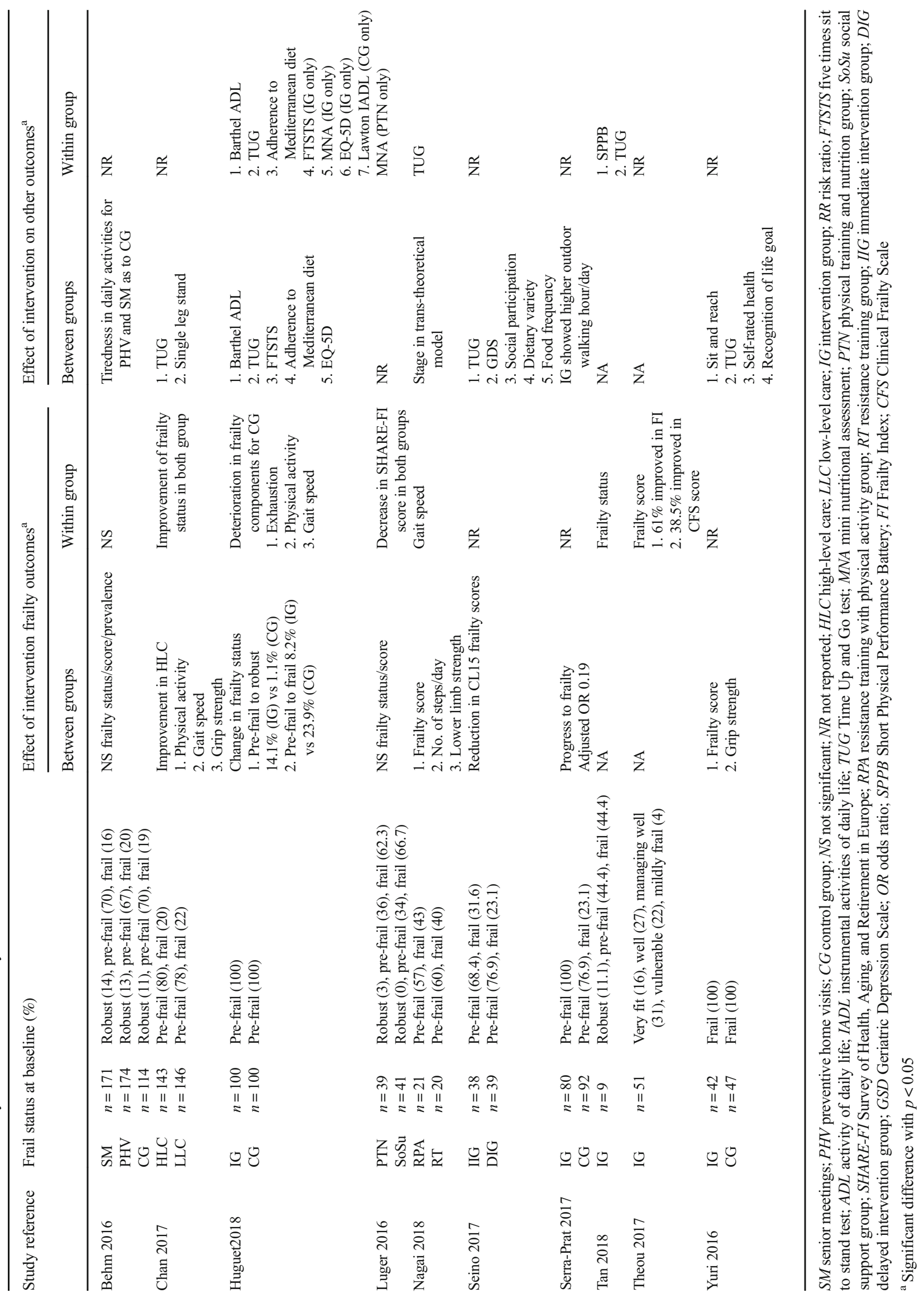




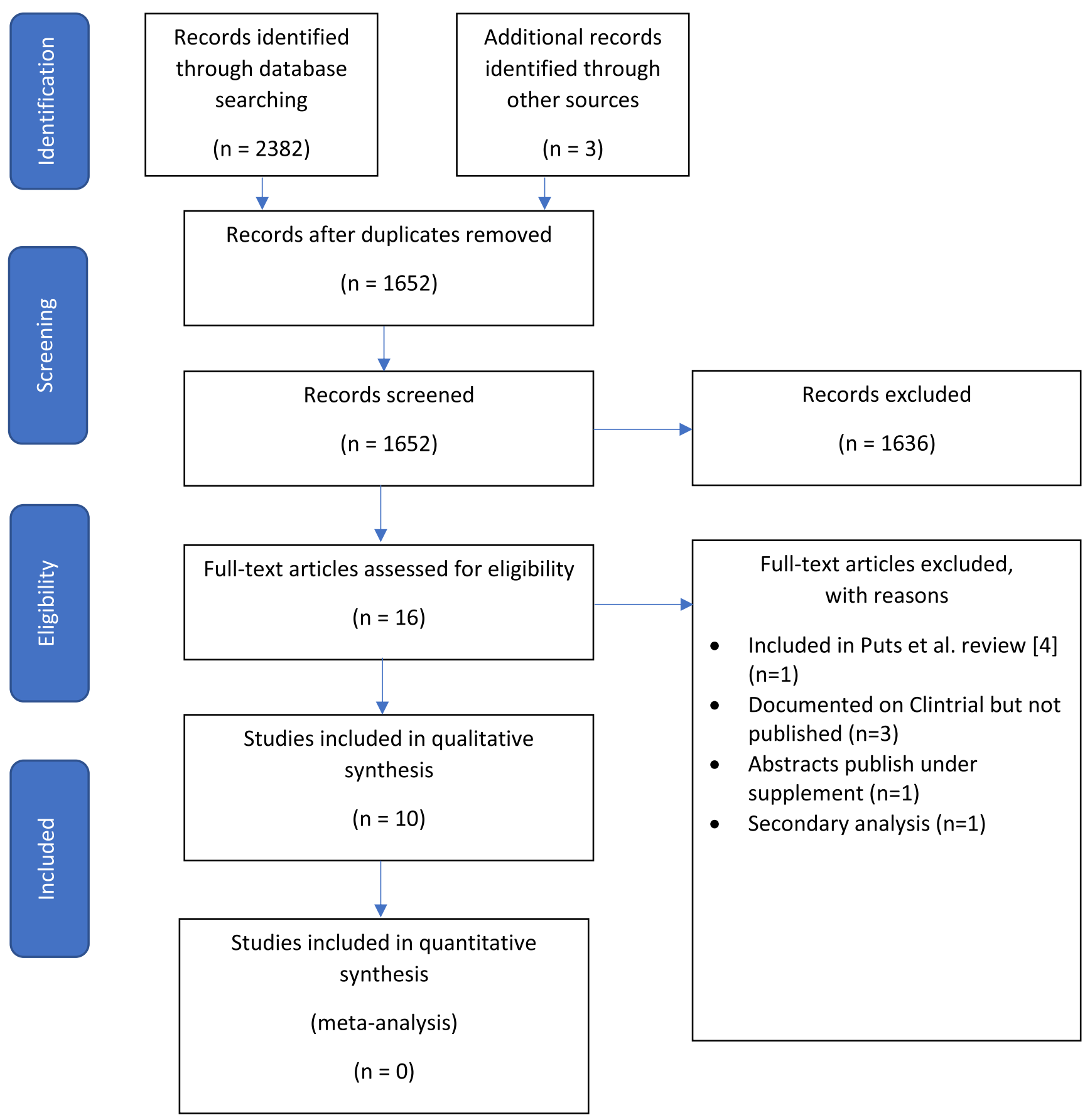

Fig. 1 PRISMA flow chart

dietary consultation [23-25], and nutrition education lecture and group activities [27]. Psychosocial interventions included programs on social capital, networks and hobbies [27], problem-solving therapy, and interventions on depression and emotion coping strategies [23]. Additionally, goal setting motivation strategy was used in three studies [25, 26, 31].

Apart from interventions that comprised of discrete visit/ meetings [22], intervention duration ranged from 1 month [22] to 1 year [28]. Some studies implemented the interventions using available community resources such as nonprofessional volunteers [25], social entrepreneur [29], or primary care $[28,30]$. Of the eight controlled studies, four studies [24, 27, 28, 31] reported significant improvement/ maintenance in frailty status/scores compared to control groups (Table 3). Three studies involved multi-domain (including exercise training, nutritional with/without another psycho/social component) interventions compared with nothing [27] or usual primary care [24, 28] as control. The study by 
Yuri et al. [31] compared preventive care program (exercise classes with nutrition and oral health education) coupled with life goal setting against a preventive care program alone.

\section{Effect of Interventions on Frailty}

Overall, 9 of the 10 studies [23-31] reported reduction in the level of frailty with intervention. While there was no difference in frailty status, a multi-domain intervention that included resistance exercise training reported significant improvement in frailty components of strength and gait speed compared to a single education session control [23]. Nagai et al. [26] reported greater increase in lower limb strength and daily steps with significant reductions in frailty scores in the intervention than control group.

However, preventive home visits or senior group meetings involving health and social information and education were not effective in reducing frailty as measured by sum of frailty indicators, even though "tiredness in daily activities" was reduced [22]. Although Luger et al. [25] reported no betweengroup differences, both groups experienced reduced SHAREFI frailty score. Reduction in frailty scores was identified in both single-arm studies $[29,30]$ and may be attributed to improvement in strength and muscular endurance with functional power training [29], and multi-disciplinary primary care with health coaching [30].

Interventions that reversed/reduced frailty included resistance, power/strength training alone [26, 29], or as part of an exercise program that included aerobic, balance, and/or coordination training, coupled with nutritional education/ intervention [23-25, 27, 28, 31]. Other effective intervention components could also contribute to frailty score reduction, either alone [25] or as part of a multi-domain/disciplinary intervention $[27,31]$. These included cognitive training with social contact [25], psychosocial/life goal support program $[27,31]$, and possibly multi-disciplinary primary care addressing polypharmacy [24] or with health coaching [30].

\section{Nutritional Interventions and Interventions' Effect on Quality of Life}

Six studies employed nutrition-related interventions [23-25, $27,28,31$ ] and seven measured interventions' effect on quality of life (QoL) [22-24, 26-28, 31]. The nutritional interventions employed included nutrition assessment [28], group nutrition education [23, 24, 27, 31], individual counseling [24], and recipe guide and/or practical sessions [25, 27].

Three $[24,25,28]$ of four $[24,25,27,28]$ studies that reported outcomes on nutritional status employed the mininutrition assessment. In these three studies [24, 25, 28], there was no difference between groups for change in nutritional status, even though there may have been increased adherence to prescribed diet [24]. This could be due to ceiling effect, as
$50-70 \%$ of the participants were well-nourished at baseline. Seino et al. [27] reported that nutrition education and practical sessions improved quality and quantity of food intake which was maintained 3 months post-intervention. Lastly, while the intervention by Yuri et al. [31] included education on nourishment, nutrition outcome was not reported.

Three $[24,27,31]$ of the five $[24,26-28,31]$ studies which measured QoL reported significant improvements post-intervention (Table 3). These studies involved group exercise and nutrition classes, in conjunction with additional psychosocial intervention. Participants who engaged in a psychosocial program [27] to improve social capital by sharing experiences, hobbies, and interests had lower geriatric depression scale scores that were maintained at 6 months follow-up. Additionally, participants who received home-based support from social workers [24] reported better EQ-5D scores. A study that employed a thorough life goal-setting program resulted in QoL improvement [31]. Conversely, participants who underwent physical training program alone [26] did not show any improvement in the SF-8 health-related QoL. Similarly, non-group home-based exercise and nutritional intervention [28] did not modify QoL.

\section{Adherence and Adverse Events}

Adherence rates to the interventions ranging from 47.5 [28] to $90.4 \%$ [27] were reported in 7 of the 10 studies [23-29] (Table 2). Home-based interventions by Chan et al. [23] reported that only $67 \%$ of participants attended at least $50 \%$ of the intervention program, while $47.5 \%$ of participants in another study [28] completed $\geq$ $70 \%$ of the recommended exercise sessions. Contrarily, the addition of social support as part of the intervention attained better adherences between 70 [25] and 90.4\% [27]. No adverse event related to study interventions was reported.

\section{Dissemination or Implementation of Interventions and Population Strategies}

The World Report on Aging and Health by WHO [10] defines "healthy aging" as the process of developing and maintaining the functional ability that enables well-being in older age. Puts et al. [6••] reported diverse policies in Europe to prevent or reduce frailty including (i) a large clinical trial SPRINTT [32], (ii) the use of SHARE-FI [33] to identify frailty at primary care, and (iii) European Commission initiative towards a systematic and integrated approach for routine screening and management of frailty through multi-modal interventions (European Scaling-up Strategy in Active and Healthy Aging) [34]. More recently, the European Union further 
launched the ADVANTAGE Joint Action [35], where member states disseminated a common directive to combat frailty [36]. There have been some policy and funding efforts in Singapore to translate research to wider health and social service practice and programs to systematically screen for and prevent/delay frailty [37]. The appreciation by Singapore health ministry officials on the prevalence and effects of frailty resulted in health and social care systems level restructuring to address the rising prevalence of frail older adults [38]. Multiple community-based programs from physical activity to multi-domain interventions have been initiated [39], though comprehensive evaluation on implementation and effectiveness of these programs are needed. Nonetheless, there is some consensus in the research, practice, and policy communities on the identification of, and intervention on frailty to maintain function and delay need for disability support [35]. Other gray literature mainly reports supportive services for disabilities rather than preventive programs or initiatives to reduce frailty and vulnerability [40, 41] (see Appendix B, Table F).

\section{Stakeholder Consultation}

While there has been an increase in the number of frailtyrelated studies over the last 10 years, with a comprehensive clinical practice guideline published recently [42], gray literature search revealed very limited large-scale dissemination and implementation of the evidence. The short questionnaire conducted with six leading researchers in the field highlighted several gaps. Across six different regions (China, France, Hong Kong, Spain, Taiwan, and USA), there was a lack of comprehensive efforts/policies to address the challenges of frailty on health and social services provision and financing, the promotion of relevant changes towards efficient and sustainable health and social model of care, and implementing frailty screening and high-risk population identification. Nonetheless, fellow research experts from Spain and China responded that their governments have launched more projects to support frailty research and developed a working group on frailty and falls, and training of primary care staff.

\section{Discussion}

In their earlier review, Puts et al. [6*•] concluded that nine of the 14 studies that reported interventions reduced the level of frailty. In the last two and a half years, the number of studies on intervention to reduce frailty in community-dwelling older people has increased fourfold compared to 2000-2015. In this updated review, nine [23-31] of 10 studies reported that the intervention reduced the level of frailty, strengthening the evidence base on frailty reduction in the community.

Importantly, more definitions/assessments of frailty had also been adopted. While six definitions were used in the 14 studies reviewed earlier, eight definitions were used in the 10 recent studies in our review. This can be attributed to the adoption of multi-dimensional and/or questionnaire/ checklist-type assessment [22, 27, 31] for rapid screening and identification. The Fried physical frailty phenotype [1] has been most commonly used to define frailty in the literature $[23,24,26,27]$ as well as the studies within our review. The increasing use of multi-dimensional frailty assessment is in line with emerging evidence for a multi-dimensional (physical, cognitive, social, and psychological) concept of frailty $[8$, 9•], with implications for multi-domain interventions. Four of 10 studies reviewed adopted a multi-dimensional frailty concept with corresponding multi-domain $[27,31]$ or multidisciplinary $[22,30]$ interventions. Four other studies also adopted multi-domain interventions [23-25, 28] with only two studies employing physical training alone [26, 29]. Psychosocial interventions were included in five of the studies [23, 25-27, 31], and six studies implemented at least certain interventions in a group setting [23, 24, 26, 27, 29, 31].

Puts et al. [6••] reported that effective interventions included exercise, nutrition, cognitive training, geriatric assessment, and management. The recent clinical practice guidelines for frailty strongly recommended resistance training within a physical activity program and for poly-pharmacy to be addressed by reducing inappropriate medication(s) [42]. In our review, resistance-type training was the most commonly employed intervention modality that reduced frailty. Resistance training for strength and/or power was employed alone [26, 29], or as part of an exercise program that also included aerobic, balance, and/or coordination training $[23-28,31]$ reversed/reduced frailty. No adverse event related to the interventions was reported, suggesting these training to be safe. This confirmed the benefits of resistance training in frail adults including those above 80 years old.

Physical frailty is prevalent among malnourished older adults, and poor nutrition is also an underlying cause of frailty $[43,44]$. Furthermore, adverse health outcomes attributed to poor nutrition are likely to be associated with physical frailty [45]. In addition to physical training, nutritional education/ intervention had also been increasingly employed within effective multi-domain interventions [23-25, 27, 28, 31]. Rather than providing pre-packed meals or food supplements, reviewed studies employed a combination of more realistic and sustainable nutritional interventions suited to community-dwelling older adults-nutrition assessment, group nutrition education, individual counseling, recipe guide, and/or practical sessions. This adds to the body of knowledge on appropriate interventions for community-dwelling prefrail/frail older persons with poor nutrition. While not tested 
in any of the reviewed studies for frail older adults but relevant to those at nutritional risk, community kitchens have been implemented as a public health strategy to prevent food insecurity through reducing social isolation, improving food and cooking skills and self-empowerment [46]. Therefore, we recommend that this needs study for application to communitydwelling frail older adults who are at nutritional risk. Other intervention components also contributed to frailty reduction, either alone [25] or as part of a multi-domain/disciplinary intervention [24, 27, 31]. These included cognitive training with social contact [25], psychosocial/life goal support program [27, 31], and possibly multi-disciplinary primary care with health coaching [30] or addressing poly-pharmacy [24].

It is interesting to note that prevalence of frailty decreased to the same extent for groups that received volunteer administered (i) home-based physical training and nutrition program $(-17 \%)$ or (ii) cognitive training and social support $(-16 \%)$ [25]. The employment of robust older people as volunteers could potentially encourage community empowerment and contribute to a sustainable, positively influential health intervention. Our review found more recent research that studied the implementation of the interventions employing available community resources such as nonprofessional volunteers [25], social enterprise [29], or primary care [28, 30]. These are relevant for community translation. There is a definite need for large-scale, well-designed RCTs to evaluate a paradigm of frailty management embedded as a new element in existing service structures which systematically identify frail older adults (and their caregivers as appropriate) for targeted management which include nutritional and physical and psychosocial interventions and individualized support and training, aimed at maximizing function and promoting aging in place. There is also a need for more implementation research on the effect role of primary care in frailty screening and management vis-à-vis other community service providers.

\section{Community Translation}

For successful translation from research to practice, interventions should be effective in reducing frailty and well-adopted by the frail older adults [47] so as to achieve sustainable improvement in health. A multi-domain intervention for frail older people was more than twice as effective among those who are compliant [48]. Low adherence to interventions can pose significant challenge to effective translation. Most of the studies reviewed employed psychosocial interventions (e.g., addressing goal setting, self-efficacy, or enhancing social capital) $[23,25-27,31]$ and/or implemented at least certain interventions in a group setting [23, 24, 26, 27, 29, 31]. These studies reported adherence rates of at least $50-60 \%$ [23, 24] to more than $85 \%$ [25-27, 29], suggesting that psychosocial well-being improved participation in exercise, nutrition, and other self-management interventions to reduce frailty. This highlights the importance of motivation and social interactions in well-designed community programs, which can sustain participation and effectiveness of programs to reduce physical and social frailty and maintain function. Of note, social frailty [49] has been shown to predict adverse health and disability in community-dwelling older adults [50, 51].

There is a lack of published evidence on the benefits of programs that focused specifically on community translation. Nonetheless, the State of the Art Report (SoAR) [36] recommended the use of Comprehensive Geriatric Assessment (CGA) to assess and manage older people and considerations for three dimensions (physical, cognitive, and psychosocial) of frailty. General Practitioners (GPs) were identified as preferred healthcare professionals to screen and monitor for frailty in the primary care settings. Nonetheless, an integrated, multi-disciplinary model of management [52] comprising a mix of primary care clinicians [22, 24, 28, 30, 31], social workers [24], caregivers, and trained volunteers/buddies [25] may be necessary for sustainable implementation of interventions without significant additional resource demands for the routine management of frail older people. In different developed countries, there is still a lack of comprehensive efforts/ policies to address the challenges of frailty on health and social services provision and financing, the promotion of relevant changes towards efficient and sustainable health and social model of care, and implementing frailty screening and high-risk population identification. Nonetheless, there is an increasing awareness on the need to address frailty, and a few governments have launched specific initiatives to support frailty research and implementation in parts of their healthcare system.

\section{Strengths and Limitations of Study}

To our knowledge, this is the first review article to consider community translation of interventions to reduce frailty. We had restricted our search to studies with frailty measurements before and after the intervention, and excluded studies that targeted frail elderly that examine specific domains of physical, cognitive, nutritional, or social without specifically listing frailty as an outcome. With the inclusion of more such studies, the evidence on effectiveness of multi-domain interventions would probably have been stronger. The studies in our review also lacked assessments and interventions (with exception of Luger et al. [25]) on the cognitive domain of frailty. Nonetheless, while there are many studies employing dual-tasking training interventions or addressing cognitive decline in the literature that are not classified under frailty intervention and are not within the scope this review, in conjunction with multi-domain interventions, there should be adequate 
assessments specific to each domain so as to provide a holistic assessment of frailty [53, 54]. The quality of future studies can be improved with better-powered randomized controlled trials, with study sample that is representative of the study population and assessors who are blinded to intervention assessments. Furthermore, evaluation on the cost-effectiveness of the intervention should be performed to provide better informed wider implementation of the program. Lastly, the inconsistent definitions and measurements of frailty have been recognized within this field [55], limiting comparisons between components of the multi-domain interventions and its effect on frailty.

\section{Conclusion}

Updating previous findings of 14 studies from 2000 to 2015 by Puts et al. [6••], we reviewed 10 additional studies from 2016 to 2018 and found that up to date, in 18 of 24 studies overall, interventions were effective in preventing or reducing frailty (as the intervention target) in community-dwelling older adults. Multi-dimensional frailty definitions and a multi-domain approach have been increasingly employed for frailty identification and intervention. The effective interventions included various modalities of exercise training, nutrition education/program, cognitive training, geriatric assessment, and management coupled with psychosocial interventions/support that may sustain program participation. While resistance training was the most commonly employed modality of physical training in our review, we did not find evidence that it is more effective than other modalities. Effective interventions can be delivered in a primary care or other community settings with a multi-disciplinary combination of professionals and volunteers. There has been better recognition of the need for community translation for frailty reduction in the developed countries, with initial policy efforts introduced in some countries. However, more comprehensive policies are needed to address frailty prevention/reduction through appropriate health and social programs/services provision and financing, the promotion of relevant changes towards efficient and sustainable health and social model of care, and implementing frailty screening and high-risk population identification and management.

\section{Compliance with Ethical Standards}

Conflict of Interest Xiao Liu, Daniella Hui-Min Ng, Jean Wei-Ting Seah, Yasmin Lynda Munro, and Shiou-Liang Wee declare that they have no conflict of interest.

Human and Animal Rights and Informed Consent This article does not contain any studies with human or animal subjects performed by any of the authors.
Open Access This article is distributed under the terms of the Creative Commons Attribution 4.0 International License (http:// creativecommons.org/licenses/by/4.0/), which permits unrestricted use, distribution, and reproduction in any medium, provided you give appropriate credit to the original author(s) and the source, provide a link to the Creative Commons license, and indicate if changes were made.

\section{References}

Papers of particular interest, published recently, have been highlighted as:

- Of importance

•- Of major importance

1. Fried LP, Tangen CM, Walston J, Newman AB, Hirsch C, Gottdiener J, et al. Frailty in older adults: evidence for a phenotype. J Gerontol Ser A: Biol Sci. 2001;56(3):M146-M57.

2. Fried TR, Mor V. Frailty and hospitalization of long-term stay nursing home residents. J Am Geriatr Soc. 1997;45(3):265-9.

3. Santos-Eggimann B, Cuénoud P, Spagnoli J, Junod J. Prevalence of frailty in middle-aged and older community-dwelling Europeans living in 10 countries. J Gerontol. 2009;64(6):675-81.

4. Collard RM, Boter H, Schoevers RA, Voshaar RCO. Prevalence of frailty in community-dwelling older persons: a systematic review. J Am Geriatr Soc. 2012;60(8):1487-92.

5. Siriwardhana DD, Hardoon S, Rait G, Weerasinghe MC, Walters KR. Prevalence of frailty and prefrailty among communitydwelling older adults in low-income and middle-income countries: a systematic review and meta-analysis. BMJ Open. 2018;8(3): e018195.

6.• Puts MTE, Toubasi S, Andrew MK, Ashe MC, Ploeg J, Atkinson E, et al. Interventions to prevent or reduce the level of frailty in community-dwelling older adults: a scoping review of the literature and international policies. Age Ageing. 2017;46(3):383-92. This study provided a thorough scoping review on interventions to prevent or reduce frailty in community-dwelling older adults from January 2000 to February 2016. Our current review is an extention to this study.

7. Apóstolo J, Cooke R, Bobrowicz-Campos E, Santana S, Marcucci M, Cano A, et al. Effectiveness of interventions to prevent prefrailty and frailty progression in older adults: a systematic review. JBI Database System Rev Implement Rep. 2018;16(1):140. This study reviewed 21 studies from January 2001 to November 2015 that included community-dwelling older, with the addition of studies from hospitals and eldercare institutions.

8. Robbert JJG, Marcel ALM vA, Katrien GL, Jos MGAS. The predictive validity of the Tilburg Frailty Indicator: disability, health care utilization, and quality of life in a population at risk. Gerontologist. 2012;52(5):619-31.

9. Junius-Walker U, Onder G, Soleymani D, Wiese B, Albaina O, Bernabei R, et al. The essence of frailty: a systematic review and qualitative synthesis on frailty concepts and definitions. Eur J Intern Med. 2018. This study highlighted the emerging evidence for multi-dimensional concept of frailty with implications for multi-domain type of interventions for frailty.

10. World Health Organisation. World report on ageing and health. 2015. http://www.who.int/ageing/publications/world-report-2015/ en. Accessed 15 November 2018.

11. Frost R, Belk C, Jovicic A, Ricciardi F, Kharicha K, Gardner B, et al. Health promotion interventions for community-dwelling older people with mild or pre-frailty: a systematic review and meta-analysis. BMC Geriatr. 2017;17(1):157. This study reviewed 10 
studies from January 1990 to May 2016 on randomised controlled trials and crossover trials of health promotion interventions for community-dwelling older people with mild/prefrailty.

12. Arksey H, O'Malley L. Scoping studies: towards a methodological framework. Int J Soc Res Methodol. 2005;8(1):19-32.

13. Colquhoun HL, Levac D, O'Brien KK, Straus S, Tricco AC, Perrier $\mathrm{L}$, et al. Scoping reviews: time for clarity in definition, methods, and reporting. J Clin Epidemiol. 2014;67(12):1291-4.

14. Moher D, Liberati A, Tetzlaff J, Altman DG. Preferred reporting items for systematic reviews and meta-analyses: the PRISMA statement. Int J Surg. 2010;8(5):336-41.

15. New York Academy of Medicine Grey Literature Report. Grey Literature Report. http://www.greylit.org/. Accessed 15 August 2018.

16. Open Grey. System for information on grey literature. http://www. opengrey.eu/. Accessed 15 Aug 2018.

17. Science.gov. Your gateway to U. S Federal Science. https://www. science.gov/. Accessed 15 August 2018.

18. International China Conference on Frailty. 2018. https://frailtychina.com/programme.pdf. Accessed 3 September 2018.

19. Covidence [Computer program]. Veritas health innovation, Melbourne. www.covidence.org. Accessed 10 June 2018.

20. Daudt HM, van Mossel C, Scott SJ. Enhancing the scoping study methodology: a large, inter-professional team's experience with Arksey and O'Malley's framework. BMC Med Res Methodol. 2013;13(1):48.

21. Hong QN, Pluye P, Fabregues S, Bartlett G, Boardman F, Cargo M, et al. Mixed methods appraisal tool (MMAT). In: Version, vol. 2018. Canada: Canadian Intellectual Property Office; 2018.

22. Behm L, Eklund K, Wilhelmson K, Zidén L, Gustafsson S, Falk K, et al. Health promotion can postpone frailty: results from the RCT elderly persons in the risk zone. Public Health Nurs. 2016;33(4): 303-15.

23. Chan D-CD, Tsou H-H, Chang C-B, Yang R-S, Tsauo J-Y, Chen C$\mathrm{Y}$, et al. Integrated care for geriatric frailty and sarcopenia: a randomized control trial. J Cachexia Sarcopenia Muscle. 2017;8(1):78.

24. Gené Huguet L, Navarro González M, Kostov B, Ortega Carmona M, Colungo Francia C, Carpallo Nieto M, et al. Pre frail 80: multifactorial intervention to prevent progression of pre-frailty to frailty in the elderly. J Nutr Health Aging. 2018:1-9.

25. Luger E, Dorner TE, Haider S, Kapan A, Lackinger C, Schindler K. Effects of a home-based and volunteer-administered physical training, nutritional, and social support program on malnutrition and frailty in older persons: a randomized controlled trial. J Am Med Dir Assoc. 2016;17(7):671-e9.

26. Nagai K, Miyamato T, Okamae A, Tamaki A, Fujioka H, Wada Y, et al. Physical activity combined with resistance training reduces symptoms of frailty in older adults: a randomized controlled trial. Arch Gerontol Geriatr. 2018;76:41-7.

27. Seino S, Nishi M, Murayama H, Narita M, Yokoyama Y, Nofuji Y, et al. Effects of a multifactorial intervention comprising resistance exercise, nutritional and psychosocial programs on frailty and functional health in community-dwelling older adults: a randomized, controlled, cross-over trial. Geriatr Gerontol Int. 2017;17(11): 2034-45.

28. Serra-Prat M, Sist X, Domenich R, Jurado L, Saiz A, Roces A, et al. Effectiveness of an intervention to prevent frailty in pre-frail community-dwelling older people consulting in primary care: a randomised controlled trial. Age Ageing. 2017;46(3):401.

29. Tan QLL, Chye LMY, Ng DHM, Chong MS, Ng TP, Wee SL. Feasibility of a community-based functional power training program for older adults. Clin Interv Aging. 2018;13:309-16.

30. Theou O, Park GH, Garm A, Song X, Clarke B, Rockwood K. Reversing frailty levels in primary care using the CARES model. Can Geriatr J. 2017;20(3):105-11.
31. Yuri Y, Takabatake S, Nishikawa T, Oka M, Fujiwara T. The effects of a life goal-setting technique in a preventive care program for frail community-dwelling older people: a cluster nonrandomized controlled trial. BMC Geriatr. 2016;16(1):101.

32. Sprintt. Sarcopenia and physical frailty in older people. http://www. mysprintt.eu/en. Accessed 24 July 2018.

33. Romero-Ortuno R, Walsh CD, Lawlor BA, Frailty KRAA. Instrument for primary care: findings from the Survey of Health, Ageing and Retirement in Europe (SHARE). BMC Geriatr. 2010;10(1):57.

34. European Commision. European scaling-up strategy in active and healthy ageing. 2015. https://ec.europa.eu/research/innovationunion/pdf/active-healthy-ageing/scaling_up_strategy.pdf. Accessed 28 July 2018.

35. Rodríguez Mañas L, García-Sánchez I, Hendry A, Bernabei R, Roller-Wirnsberger R, Gabrovec B, et al. Key messages for a frailty prevention and management policy in Europe from the Advantage Joint Action Consortium. J Nutr Health Aging. 2018;22(8):892-7.

36. Rodríguez-Laso Á, Ángeles M, Mora C, Sánchez IG, Rodríguez ML, Bernabei R et al. State of the art report on the prevention and management of frailty. http://advantageja.eu/images/SoARAdvantageJA Fulltext.pdf. Accessed 21 August 2018.

37. Wee SL. Translation of research on frailty identification, prevention and care into action - Singapore perspective. J Frailty Aging. 2018;7.

38. Lim W, Wong S, Leong I, Choo P, Pang W. Forging a frailty-ready healthcare system to meet population ageing. Int J Environ Res Public Health. 2017;14(12):1448.

39. Chen CY, Gan P, How CH. Approach to frailty in the elderly in primary care and the community. Singap Med J. 2018;59(5):240-5.

40. Hostetter M, Klein S, McCarthy D. Aging gracefully: the PACE approach to caring for frail elders in the community. 2016. http:// www.greylit.org/sites/default/files/collected files/2016-08/1893 Hostetter_PACE_OnLok_case_study.pdf. Accessed 8 October 2018.

41. British Columbia - Ministry of Health. An Action Plan to Strengthen Home and Community Care for Seniors. 2017. https:// www.health.gov.bc.ca/library/publications/year/2017/home-andcommunity-care-action-plan.pdf. Accessed 16 August 2018.

42. Dent E, Lien C, Lim WS, Wong WC, Wong CH, Ng TP, et al. The Asia-Pacific clinical practice guidelines for the management of frailty. J Am Med Dir Assoc. 2017;18(7):564-75.

43. Verlaan S, Ligthart-Melis GC, Wijers SLJ, Cederholm T, Maier A, de van der Schueren MAE, et al. High prevalence of physical frailty among community-dwelling malnourished older adults-a systematic review and meta-analysis. J Am Med Dir Assoc. 2017;18(5): 374-81.

44. Wei K, Nyunt MSZ, Gao Q, Wee SL, Ng T-P. Frailty and malnutrition: related and distinct syndrome prevalence and association among community-dwelling older adults: Singapore longitudinal ageing studies. J Am Med Dir Assoc. 2017;18(12):1019-28.

45. Wei K, Nyunt M, Gao Q, Wee S, Yap K, Ng T. Association of frailty and malnutrition with long-term functional and mortality outcomes among community-dwelling older adults: results from the Singapore longitudinal aging study 1. JAMA Netw Open. 2018;1(3):e180650.

46. Iacovou M, Pattieson DC, Truby H, Palermo C. Social health and nutrition impacts of community kitchens: a systematic review. Public Health Nutr. 2012;16(3):1-9.

47. Grimshaw JM, Eccles MP, Lavis JN, Hill SJ, Squires JE. Knowledge translation of research findings. Implement Sci. 2012;7(1):50.

48. Fairhall N, Sherrington C, Cameron ID, Kurrle SE, Lord SR, Lockwood $\mathrm{K}$, et al. A multifactorial intervention for frail older people is more than twice as effective among those who are 
compliant: complier average causal effect analysis of a randomised trial. J Phys. 2016;63(1):40-4.

49. Bunt S, Steverink N, Olthof J, van der Schans CP, Hobbelen JSM. Social frailty in older adults: a scoping review. Eur J Ageing. 2017;14(3):323-34.

50. Makizako H, Shimada H, Tsutsumimoto K, Lee S, Doi T, Nakakubo S, et al. Social frailty in community-dwelling older adults as a risk factor for disability. J Am Med Dir Assoc. 2015;16(11)

51. Teo NB, Gao QP, Nyunt MSZP, Wee SLP, Ng T-PMD. Social Frailty and Functional Disability: Findings from the Singapore longitudinal ageing studies. J Am Med Dir Assoc. 2017;18(7): 637.e13-9.

52. Clarfield AM, Bergman H, Kane R. Fragmentation of care for frail older people-an international problem. Experience from three coun- tries: Israel, Canada, and the United States. J Am Geriatr Soc. 2001;49(12):1714.

53. Dent E, Kowal P, Hoogendijk EO. Frailty measurement in research and clinical practice: a review. Eur J Intern Med. 2016;31:3-10.

54. Rockwood K, Stolee P, Fox PRA. Use of goal attainment scaling in measuring clinically important change in the frail elderly. J Clin Epidemiol. 1993;46(10):1113-8.

55. Bouillon K, Kivimaki M, Hamer M, Sabia S, Fransson EI, SinghManoux A, et al. Measures of frailty in population-based studies: an overview. BMC Geriatr. 2013;13:64.

Publisher's Note Springer Nature remains neutral with regard to jurisdictional claims in published maps and institutional affiliations. 